\section{REVISTA BRASILEIRA DE QUALIDADE DE VIDA}

\title{
A influência da prática do goalball e da deficiência na percepção da qualidade de vida de pessoas com deficiência visual
}

\section{The influence of goalball and disability in the perception of quality of life of people with visual impairment}

\author{
Sabrina Schröder \\ Faculdades Integradas de Itararé - FAFIT - Itararé - São Paulo - Brasil \\ leandro.vargas@uol.com \\ José Roberto Herrera Cantorani \\ Faculdades Integradas de Itararé - FAFIT - Itararé - São Paulo - Brasil \\ cantorani@yahoo.com \\ Leandro Martinez Vargas \\ Faculdades Integradas de Itararé - FAFIT - Itararé - São Paulo - Brasil \\ leandro.vargas@uol.com.br \\ Thaiane Moleta Vargas \\ Universidade Estadual de Ponta Grossa - UEPG - Ponta Grossa - Paraná - Brasil \\ thaiane_moleta@yahoo.com
}

\section{RESUMO}

OBJETIVO: Analisar a influência da prática sistemática e regular de goalball e da deficiência na percepção da qualidade de vida (QV) de pessoas com deficiência visual (PCDv).

MÉTODOS: Participaram da pesquisa 15 pessoas com deficiência visual, de ambos os sexos, dentre os quais 10 praticam o goalball de forma sistematizada e 5 não praticam nenhum tipo de atividade física sistematizada. A percepção da QV foi avaliada por meio do instrumento WHOQOL-DIS-PD. A análise dos dados foi realizada por meio da planilha desenvolvida por Pedroso et al. (2010), a qual utiliza a plataforma do Office Excel da Microsoft ${ }^{\circledR}$.

RESULTADOS: Os resultados oriundos do WHOQOL-DIS-PD evidenciaram uma discreta melhor percepção da QV entre as PCDv que praticam o goalball em comparação ao grupo que não pratica. A questão que mais se destacou a favor dos praticantes de goalball foi a relacionada à discriminação. A favor dos não praticantes de atividade física, a questão relacionada ao impacto da deficiência foi mais bem percebida.

CONCLUSÃO: O estudo apresenta indícios de que a percepção da QV como um todo e algumas facetas e domínios que compõem o WHOQOL-DIS-PD podem ser influenciadas pela adesão à prática sistemática do goalball.

PALAVRAS-CHAVE: Qualidade de vida. Goalball. Deficiência visual. 


\section{ABSTRACT}

OBJECTIVE: To analyze the influence of systematic and regular practice of goalball and disability in the perception of quality of life (QOL) of people with visual impairment (PVI).

METHODS: 15 visually impaired people participated on study, of both sexes, including 10 who practice organized goalball and 5 who do not practice any kind of systematic physical activity. The perception of QoL was assessed by the WHOQOL-DIS-PD instrument. Data analysis was performed using the worksheet developed by Pedroso et al. (2010), which uses the Microsoft® Office Excel platform.

RESULTS: The results derived from the WHOQOL-DIS-PD showed a discrete better perception of QoL among PVI who practice goalball compared to the group that does not practice. The issue that stood out in favor of goalball practitioner's was related to discrimination. In favor of non-AF practitioner's, the issue related to the impact of disability was better perceived.

CONCLUSIONS: This study presents evidence that the perception of QoL as a whole, and some facets and areas that make up the evaluation instruments, can be influenced by the practice of goalball.

KEYWORDS: Quality of life. Goalball. Visual impairment.

\section{Introdução}

Dados dos recenseamentos realizados no Brasil mostram um crescimento significativo do número de pessoas com deficiência (PCD) (CANTORANI et al., 2015). A elevação do número deuse, sobretudo, pelo avanço na forma de olhar e de entender a deficiência. As mudanças verificadas nos recenseamentos acompanharam a evolução conceitual de deficiência. Essa evolução foi ajustada mediante conquistas sociais na área do direito à vida e à igualdade de condições, que viabilizaram uma política de acessibilidade e também o interesse pela qualidade de vida (QV) das PCDs (CANTORANI et al., 2015).

O recenseamento de 2010 aponta que aproximadamente $23,9 \%$ da população brasileira apresenta pelo menos um tipo de deficiência. Comparada às demais deficiências, a visual é significativamente maior, com uma prevalência de $18,6 \%$, seguida pela motora $(7,0 \%)$, auditiva $(5,1 \%)$ e intelectual (1,4\%) (OLIVEIRA, 2012).

A deficiência visual, de acordo com Teixeira (2010, p. 01), “[...] refere-se a uma situação irreversível de diminuição da resposta visual, em virtude de causas congênitas ou hereditárias, mesmo após tratamento clínico e/ou cirúrgico e uso de óculos convencionais". Essa diminuição da resposta visual resulta em dificuldade de locomoção e de execução de tarefas motoras. Por sua vez, a falta de atividade física (AF) implica no surgimento de diversas doenças crônico-degenerativas e no distanciamento de um estilo de vida mais saudável (ZUCHETTO; CASTRO, 2002). Ciente dos benefícios oferecidos pela prática regular de $\mathrm{AF}$, diversos autores têm voltado o olhar para a quantificação do nível de AF entre as PCDs, pois a mesma tem sido um dos principais indicadores de uma boa QV (ZUCHETTO; CASTRO, 2002; NOCE; SIMIM; MELLO, 2009; SCHERER; LOPES, 2013).

Entre as atividades esportivas praticadas pelas pessoas com deficiência visual (PCDv), encontra-se o goalball, modalidade esportiva que possui adaptações que atendem suas necessidades e limitações (CONFEDERAÇÃO BRASILEIRA DE DESPORTOS DE DEFICIENTES VISUAIS, 2015). Essa modalidade, criada em 1946 na Áustria, tinha como objetivo reabilitar veteranos de guerra que perderam a visão. Atualmente, o goalball é uma modalidade exclusiva para PCDs, especialmente a visual. Uma das adaptações feitas exclusivamente para as PCDv é a colocação de um guizo no interior da bola, que emite sons e permite aos jogadores perceberem a localização e a direção da bola. 
A literatura mostra que a prática regular e sistemática de $\mathrm{AF}$ promove bem-estar físico e também proteção contra o surgimento de doenças crônicas (ZUCHETTO; CASTRO, 2002; NOCE; SIMIM; MELLO, 2009). Por esse motivo é considerada como um fator preponderante para um estilo de vida saudável (SCHERER; LOPES, 2013).

Os principais relatos sobre os efeitos benéficos da prática da AF regular para a saúde giram em torno de pesquisa realizadas como pessoas sem deficiência. Contudo, os mesmos benefícios dessa prática podem ser conquistados também pelas PCDs. Ademais, é preciso atenção ao fato de que as PCDs tendem a ser menos ativas fisicamente (SCHEREER; LOPES, 2013).

A dificuldade que uma PCD possui para praticar AF, em especial as PCDv, é maximizada quando a maioria dos espaços destinados a tal prática não são adaptados para as suas necessidades e limitações (ZUCHETTO; CASTRO, 2002). Soma-se a esse quadro o fato das PCDv não apresentarem a mesma evolução em termos de desenvolvimento motor em comparação às pessoas sem deficiência, e, por essa razão, apresentarem na fase adulta défices de coordenação motora que envolvem as capacidades de coordenação, ritmo, esquema corporal, orientação espacial e consciência corporal (SCHERER; LOPES, 2013).

Portanto, considerando que as PCDv apresentam menores condições de praticar atividade física de forma sistemática, principalmente pela limitações e privações que o ambiente lhe impõe, e que a falta de prática de AF pode desencadear diversos problemas, incluindo a diminuição da QV, o presente estudo tem por objetivo investigar a influência do nível de $\mathrm{AF}$ e da deficiência na percepção da QV de um grupo de PCDv da cidade de Itapeva-SP.

\section{Metodologia}

O presente estudo foi desenvolvido na Associação dos Deficientes Visuais de Itapeva Luz da Visão. O período da investigação deu-se entre os meses de março e agosto de 2014. A população foi composta por 15 voluntários, todos acima de 18 anos, de ambos os sexos, com deficiência visual congênita ou adquirida.

$\mathrm{Na}$ Luz da Visão é ofertada a prática da modalidade paradesportiva goalball. Dessa forma, as PCDv pertencentes à referida instituição que praticavam sistematicamente e regularmente (mínimo duas vezes por semana) o goalball, foram enquadradas no grupo que pratica AF. Já as que não praticam nenhum tipo de AF de forma sistemática (organizada) ou o goalball de forma regular foram enquadradas no grupo de não praticantes de $\mathrm{AF}$.

Para avaliar a percepção da QV optou-se pelo WHOQOL-DIS, instrumento criado pela Organização Mundial da Saúde (THE WHOQOL GROUP, 1996) para atender as peculiaridades vivenciadas pelas PCDs. Esse instrumento foi desenvolvido em três versões: uma para pessoas com deficiência física (WHOQOL-DIS-PD), uma para pessoas com deficiência intelectual (WHOQOLDIS-ID) e uma para os responsáveis e/ou cuidadores das pessoas com deficiência intelectual (WHOQOL-DIS-ID Proxy); para os casos em que as PCDs não tenham condição de responder (THE-WHOQOL-DIS-GROUP, 2011). Para o presente estudo foi utilizado o WHOQOL-DIS-PD.

A estrutura de domínios, das facetas e do conteúdo das questões representativas de cada faceta é a mesma para as três versões. Todas as três versões constituem-se em instrumentos caracterizados pela condição de módulo adicional ao WHOQOL-bref. A estrutura desses módulos é composta por 13 facetas: uma que avalia, de modo geral, o impacto da deficiência na QV da população, e outras 12 subdivididas em 3 domínios (Quadro 1). 
Quadro 1 - Domínios e facetas do WHOQOL-DIS

\begin{tabular}{|l|l|l|}
\hline \multicolumn{1}{|c|}{ Domínios } & N & \multicolumn{1}{c|}{ Facetas } \\
\hline Geral & 1. & Impacto da deficiência. \\
\hline \multirow{2}{*}{ Domínio I - Discriminação } & 2. & Discriminação. \\
\cline { 2 - 3 } & 3. & Proteção. \\
\cline { 2 - 3 } & 4. & Perspectivas futuras. \\
\hline \multirow{2}{*}{ Domínio II - Autonomia } & 5. & Controle de sua vida. \\
\cline { 2 - 3 } & 6. & Poder de decisão. \\
\cline { 2 - 3 } & 7. & Autonomia. \\
\hline Domínio III - Inclusão & 8. & Capacidade de comunicação. \\
\cline { 2 - 3 } & 9. & Aceitação social. \\
\hline
\end{tabular}

Fonte: THE-WHOQOL-DIS-GROUP (2011).

No WHOQOL-bref existem três questões que são invertidas, são elas: 'dor e desconforto', 'dependência de medicação' e 'sentimentos negativos'. Invertidas no sentido que de que se a pessoa assinalar a pontuação mais alta nessas questões, ao invés disso ser um indício de satisfação com essas questões, como ocorre nas demais, a sensação será de que ela apresenta problemas. Logo, o percentual elevado em uma dessas questões é sinônimo de problema com as facetas relacionadas.

O mesmo ocorre no WHOQOL-DIS-PC com as questões relacionadas às facetas 'impacto da deficiência', 'discriminação', 'proteção' e 'perspectivas futuras'. Essas questões quando apresentam pontuações ou percentuais elevados mostram que o individuo apresenta insatisfação com a respectiva faceta.

Para a análise descritiva dos resultados referentes à percepção da QV pelo WHOQOL-bref, optou-se pela apresentação gráfica dos percentuais médios alcançados por ambos os grupos em cada faceta e domínio. Esses gráficos possibilitam analisar as possíveis diferenças entre os grupos de praticantes e não praticantes de $\mathrm{AF}$.

A pesquisa contou com 100,0\% da participação das PCDv regularmente inscritas na Luz da Visão. Para a aplicação dos questionários, todos os voluntários foram apresentados à proposta do estudo e o Termo de Consentimento Livre e Esclarecido (TCLE) foi lido para todos os participantes na presença da coordenadora da referida instituição. Após tomarem ciência sobre o estudo e os procedimentos de coleta dos dados, todos os participantes assinaram o TCLE.

O tratamento estatístico descritivo foi realizado por meio da planilha desenvolvida por Pedroso et al. (2010), a qual utiliza a plataforma do Office Excel da Microsoft@. O programa Statistical Package for the Social Sciences (SPSS), versão 20, foi utilizado para construir os gráficos que apresentam a distribuição dos resultados dos grupos em cada questionário.

\section{Resultados e discussão}

Os resultados apresentados referem-se às 15 PCDv participantes da pesquisa. Dessas, dez (sete homens) declararam praticar a modalidade goalball na Luz da Visão e cinco (dois homens) declararam não praticar nenhum tipo de AF sistematizada. A Tabela 1 apresenta as características da amostra por grupo:

Tabela 1 - Características gerais da amostra de PCDv da Associação dos Deficientes Visuais de Itapeva e Região - SP

\begin{tabular}{ccccccc}
\hline Grupos & $\begin{array}{c}\text { Sexo } \\
(\mathbf{n})\end{array}$ & $\begin{array}{c}\text { Idade } \\
(\text { média e DP) }\end{array}$ & $\begin{array}{c}\text { Cego } \\
(\mathbf{n})\end{array}$ & $\begin{array}{c}\text { Baixa visão } \\
(\mathbf{n})\end{array}$ & $\begin{array}{c}\text { Congênita } \\
(\mathbf{n})\end{array}$ & $\begin{array}{c}\text { Adquirida } \\
(\mathbf{n})\end{array}$ \\
\hline Praticantes de & Homens $(\mathbf{n}=\mathbf{7})$ & $44,71 \pm 13,72$ & 6 & 1 & 1 & 6 \\
Goalball & Mulheres $(\mathbf{n}=\mathbf{3})$ & $45,00 \pm 20,07$ & 2 & 1 & 0 & 3 \\
& Homens $(\mathbf{n}=\mathbf{2})$ & $43,05 \pm 6,36$ & 1 & 1 & 0 & 2 \\
Não praticam AF & Mulheres $(\mathbf{n}=\mathbf{3})$ & $48,33 \pm 24,00$ & 1 & 2 & 2 & 1 \\
& &
\end{tabular}

Fonte: Autoria própria (2015).

DP: Desvio padrão. 
Nos resultados apresentados na Tabela 1 percebe-se que, dos 15 investigados, 10 são completamente cegos e apenas 3 apresentam a deficiência visual desde o nascimento (congênita).

\subsection{Análise das facetas e domínios do WHOQOL-bref}

O Gráfico 1 apresenta o percentual médio em cada uma das facetas do questionário WHOQOL-bref:

Gráfico 1 - Percentual médio obtido pelos grupos em cada faceta do WHOQOL-bref

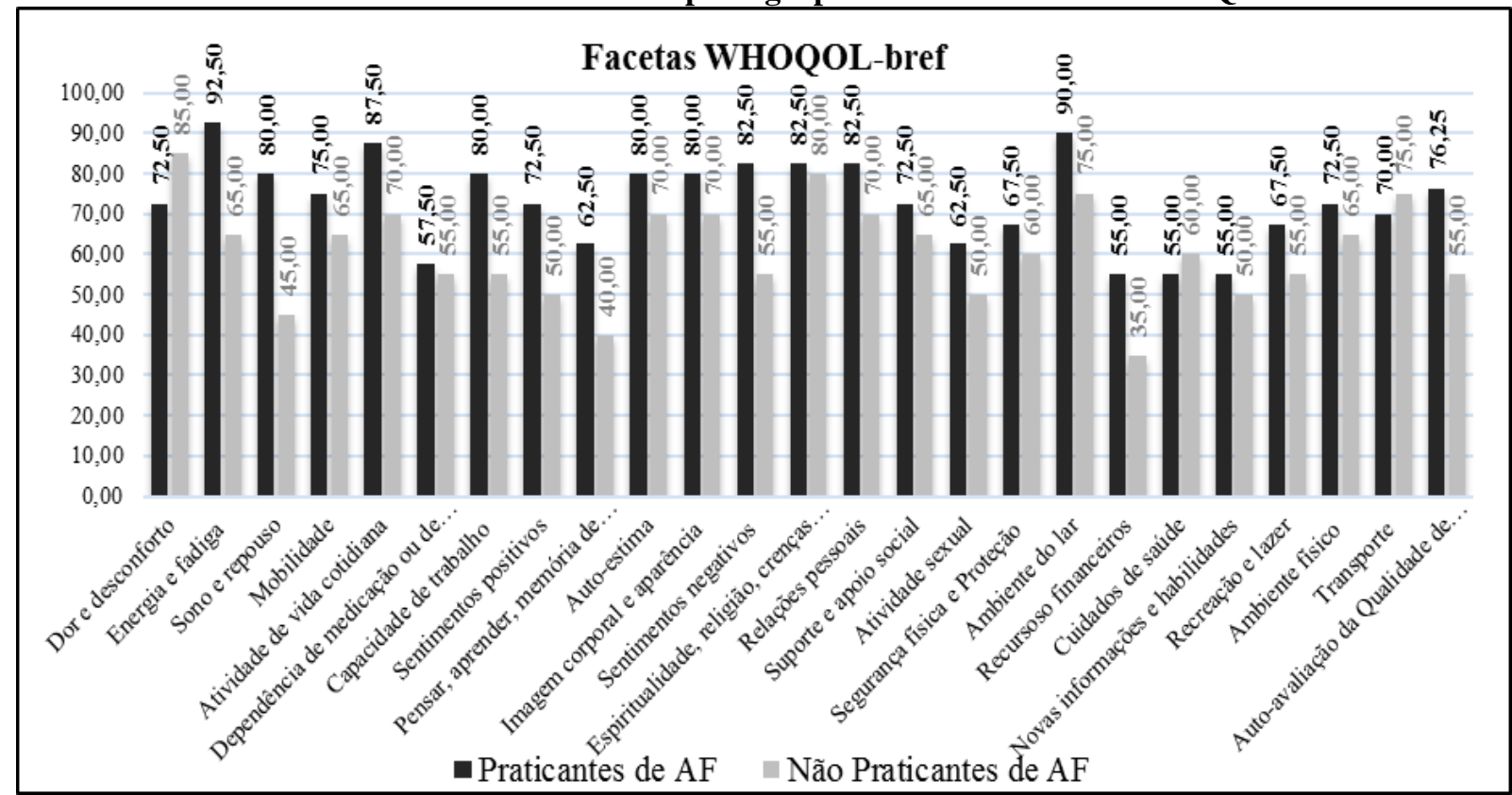

Fonte: Autoria própria (2015).

As PCDv praticantes de AF apresentaram maior percentual médio em 23 das 25 questões avaliadas pelo WHOQOL-bref. A pior percepção da QV aconteceu nas questões relacionadas à 'dor e desconforto' e 'cuidados com a saúde'.

A pior percepção da questão 'dor e desconforto' em comparação às PCDv não praticantes de $\mathrm{AF}$, possivelmente, está relacionada ao tipo de AF realizada. O goalball é um esporte que pode causar traumas osteomusculares leves tendo em vista a necessidade de o jogador ter que se lançar ao solo constantemente e manter uma posição de completa extensão corporal para defender a sua meta. Ainda, durante o ataque ao gol adversário, precisa realizar movimentos de arremesso que requerem o uso de muita potência muscular e grande amplitude articular dos ombros e quadril.

No que se refere à percepção dos 'cuidados com a saúde', é difícil apontar quais poderiam ser os possíveis fatores de influência para que as PCDv praticantes de goalball percebessem de forma negativa essa faceta em comparação ao outro grupo. Para isso, seria necessário um levantamento de questões relacionadas a essa faceta, como por exemplo, a avaliação do estado nutricional, dos hábitos alimentares, do estilo de vida e de comportamentos de risco como o uso de álcool, tabaco e outras drogas.

Nas demais facetas, os indivíduos não praticantes de $\mathrm{AF}$ apresentaram os menores percentuais, o que evidencia, para essa amostra de PCDv, que a prática regular de AF pode estar ajudando as PCDv a melhorar a sua percepção de QV.

As questões 'energia e fadiga', 'sono e repouso' e 'capacidade de trabalho' foram as mais bem percebidas entre as PCDv que declararam praticar goalball de maneira sistemática.

Sobre a 'energia e fadiga', Metzner e Fischer (2001) e Massola (2007) afirmam que a AF é um fator que está diretamente relacionado ao estilo de vida saudável, e que o mesmo pode 
condicionar a pessoa a ter mais disposição para realizar as atividades do dia-a-dia e apresentar melhor condição de suportar a fadiga em atividades extenuantes.

Em relação à questão 'sono e repouso', conforme retrata Boscolo et al. (2007), a prática regular de exercício físico pode contribuir para a $\mathrm{QV}$, proporcionando aos praticantes a melhoria em diversas áreas, além das capacidades físicas e condicionantes, como na redução da depressão e da ansiedade, na melhoria das funções cognitivas (memória, atenção e raciocínio) e da qualidade e da eficiência do sono.

A boa qualidade do sono também possui relação com a melhora dos processos de memorização e de raciocínio lógico, pois informações aprendidas são mais bem memorizadas após um período suficiente de sono (BOSCOLO et al., 2007). Pereira et al. (2010) afirmam que a qualidade do sono também está diretamente associada à satisfação com o trabalho e a produtividade.

O Gráfico 2 expõe os valores extraídos de cada um dos quatro domínios do WHOQOL-bref, no qual os indivíduos ativos apresentaram um percentual positivo nos domínios e na média total $(73,4 \%)$ em relação os indivíduos não praticantes $(60,6 \%)$

Gráfico 2 - Percentual médio obtido pelos grupos em cada domínio do WHOQOL-bref

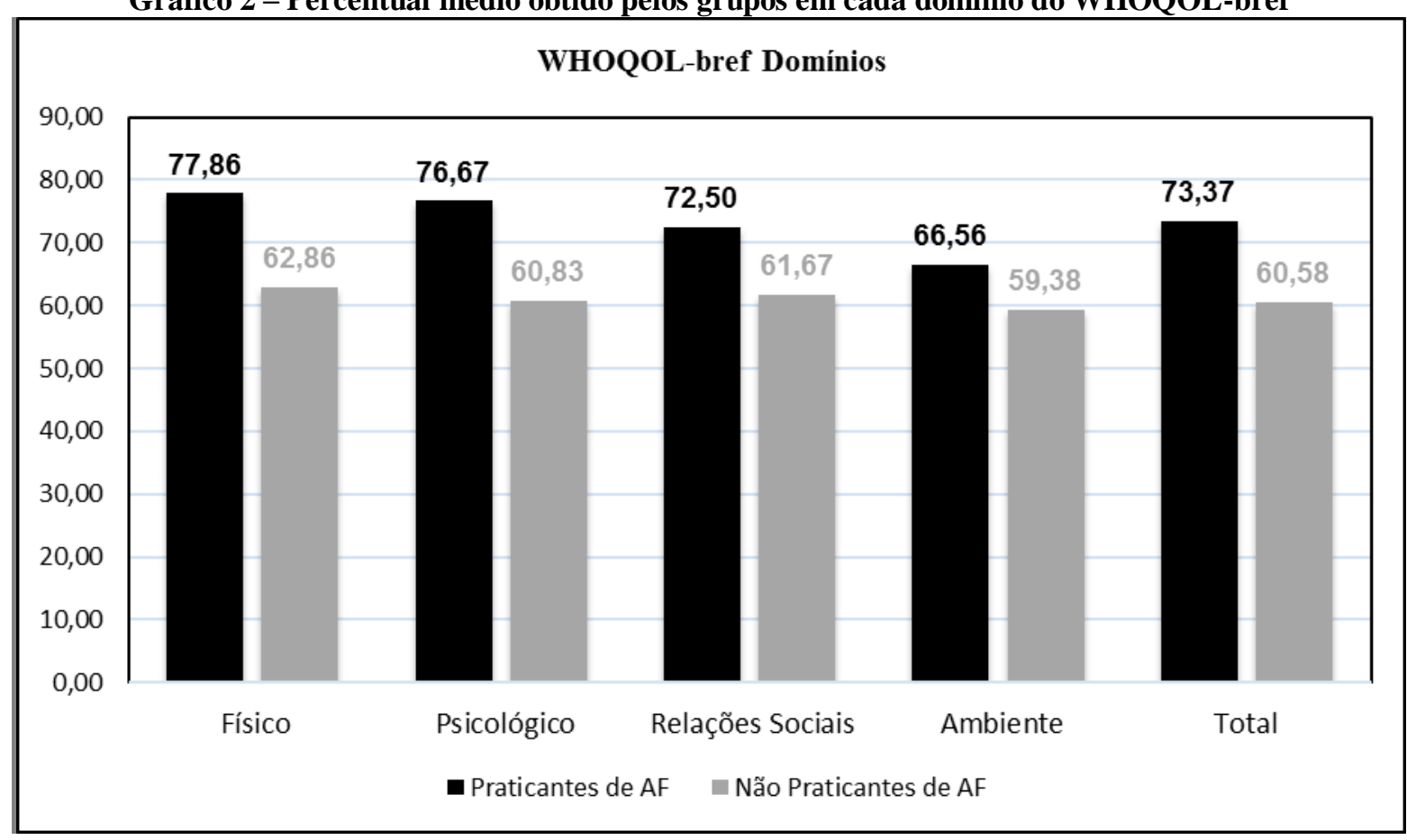

Fonte: Autoria própria (2015).

Ainda que os escores apresentem uma diferença não significativa, a percepção sobre os domínios físico e psicológico foram as que mais atraíram a atenção a favos das PCDv que praticam o goalball. Muitos estudos revelam que a AF é um fator de grande influência no bem-estar e na QV das pessoas. Principalmente a partir de momento que se considera que um dos benefícios causados pela prática regular de AF é a melhoria de fatores bio-psicossociais e culturais, e esses influenciam positivamente na percepção da QV (ZUCHETTO; CASTRO, 2002; STELLA et al., 2002).

No fator psicológico, tanto Zuchetto e Castro (2002) como Stella et al. (2002) afirmam que a prática de $\mathrm{AF}$, em particular os exercícios aeróbios, quando realizada em uma intensidade moderada por mais de 30 minutos, pode diminuir o estresse e a tensão, que são fatores que afetam negativamente $\mathrm{o}$ aspecto psicológico de um indivíduo.

Zuchetto e Castro (2002) inferem que diversos benefícios psicológicos têm sido observados em indivíduos que adotam um estilo de vida mais ativo, como: aumento do bom humor, redução do estresse, autoconceito mais positivo e percepção positiva da QV. Para Stella et al. (2002), o bom humor e a redução do estresse são produzidos pela liberação de uma grande quantidade de 
hormônios, entre eles a endorfina, responsável por atuar no sistema nervoso, fazendo com que as influências negativas do meio tenham um impacto menor sobre o indivíduo.

A diferença não significativa de percepção da QV entre os praticantes de goalball e não praticantes de AF observada na presente pesquisa é similar aos resultados apresentados por Noce, Simim e Mello (2009). Nesse estudo também não foram encontradas diferenças impactantes entre as facetas e os domínios do WHOQOL-bref. Entretanto, o grupo fisicamente ativo da pesquisa apresentou em sua totalidade valores mais positivos do que os indivíduos não praticantes, evidenciando que a prática regular de AF pode ser um fator influenciador na percepção da QV de PCDv.

\subsection{Análise das facetas e domínios do WHOQOL-DIS-PD}

Os resultados apresentados no Gráfico 3 mostram equilíbrio na maioria das facetas. O grupo de PCDv não praticantes apresentou melhor percepção em 6 das 13 facetas analisadas. A mesma quantidade de facetas foi melhor percebida pelo grupo de praticantes de AF. Em uma faceta - 'interação na sociedade' - houve igualdade.

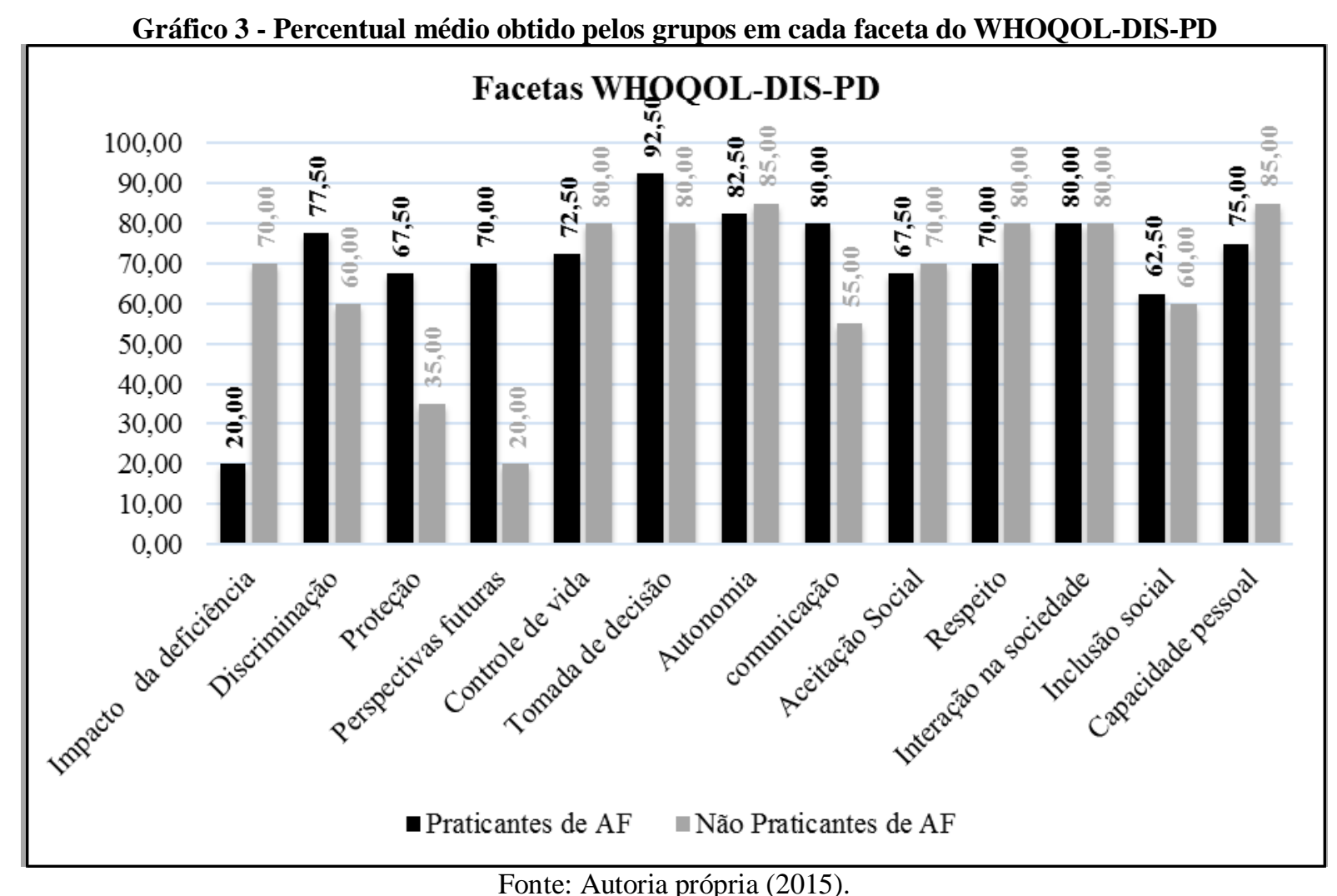

As maiores diferenças foram encontradas nas facetas 'proteção' e 'perspectivas futuras' no grupo de praticantes de goalball; e na faceta 'impacto da deficiência' no grupo dos não praticantes de AF.

No tocante a percepção sobre a 'proteção' e 'perspectivas futuras', sugere-se que a AF exerça influência significativa sobre essas duas facetas por meio da melhora da autoconfiança. Supõe-se que a prática de AF, principalmente de uma modalidade esportiva, como é o caso do goalball, implica no objetivo constante de vencer alguém. Segundo Silva e Rubio (2003), atletas nos mais diferentes níveis não apresentam somente uma preparação física distinta, mas também uma preparação mental igualmente elevada para superar os obstáculos e desafios impostos pelo adversário. Sagrar-se campeão ou vencer uma partida demanda lançar mão de vários artifícios 
mentais e psicológicos. Dessa forma, a motivação, o pensamento positivo e a autoconfiança se fazem presentes no desafio diário de superação e na maneira positiva como enxergam o futuro.

O Gráfico 4 apresenta os quatro domínios presentes no WHOQOL-DIS-PD:

Gráfico 4 - Percentual médio obtido pelos grupos em cada domínio do WHOQOL-DIS-PD

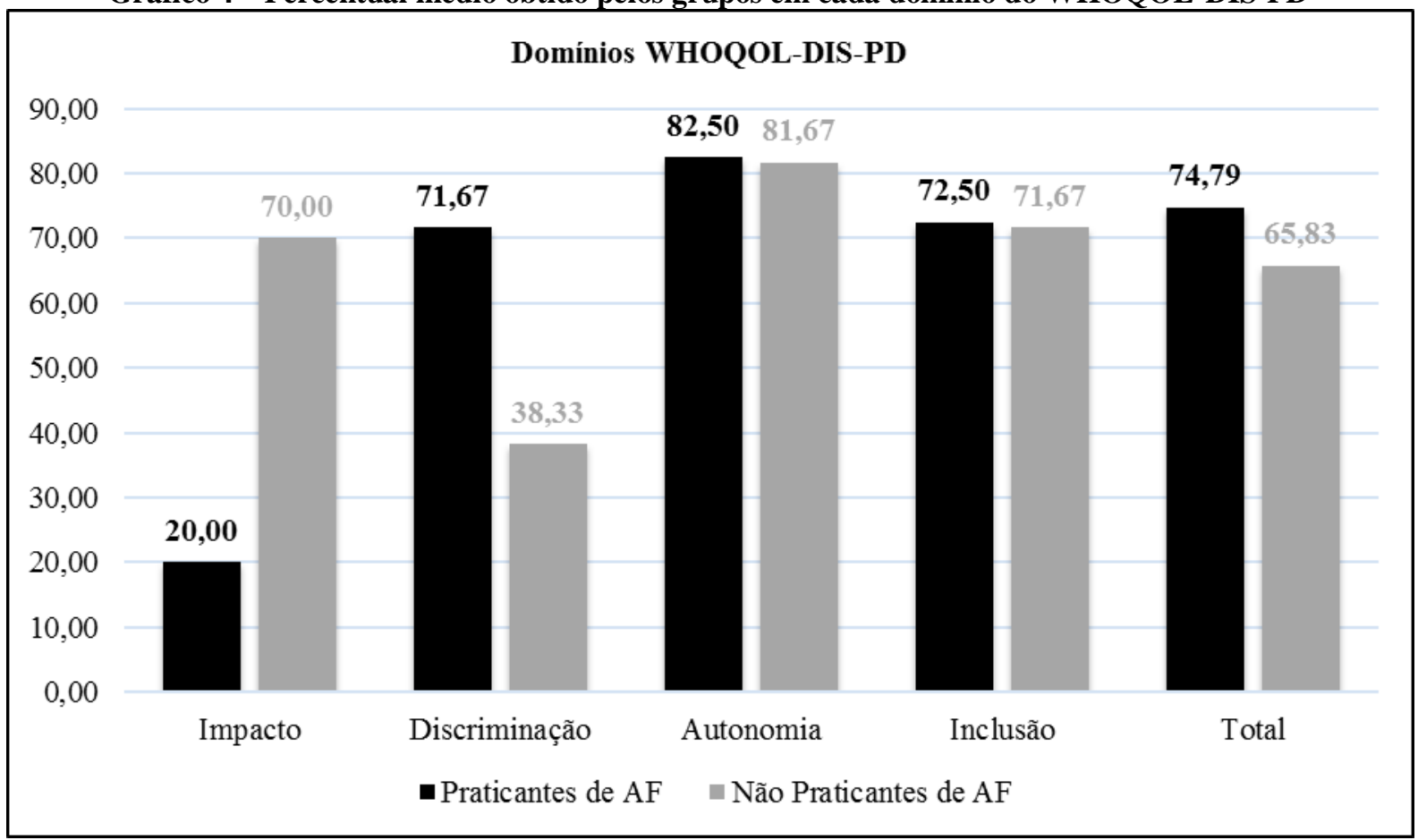

Fonte: Autoria própria (2015).

O único domínio que não sofreu alteração da média foi o relacionado ao impacto da deficiência, pois é composto por uma única faceta (Q.27). Dos escores do WHOQOL-DIS-PD, apenas os domínios relativos ao impacto da deficiência e à discriminação apresentaram diferenças consideráveis. Portanto, pôde-se observar uma influência mais negativa do impacto da deficiência sobre as PCDv praticantes de $\mathrm{AF}(20,0 \%)$, contra 70,0 \% dos não praticantes.

$\mathrm{O}$ fato das PCDv terem apresentado uma percepção negativa sobre o impacto da deficiência', em comparação ao grupo de não praticantes de AF, o presente estudo considera que a maior exposição aos meios sociais por parte das PCDv praticantes de goalball faz com que elas vivenciem um maior número de situações ambientais não adaptadas à sua condição. Por essa razão, a exposição à indivíduos não preparados para lidar com a diferença faz com que eles apresentem uma percepção negativa relacionada ao impacto da deficiência.

No entanto, essa situação de maior exposição, de viver mais as atividades do dia a dia, reflete de uma forma positiva a melhor percepção do aspecto relacionado à discriminação, pois, ao se exporem mais, os indivíduos praticantes de AF percebem serem menos discriminados em relação ao não praticantes. Esse fato se dá, provavelmente, pela naturalidade com que a sociedade os recebe de acordo com a frequência que as PCDs se envolvem naturalmente nas atividades cotidianas. Em suma, quanto mais a PCD se insere em meio à sociedade, menos problemas com a discriminação é percebida.

Entre os domínios que não apresentaram diferenças significativas, a autonomia merece destaque por retratar que, na presente amostra, a AF não exerce influência na percepção de autonomia entre as PCDv. De modo geral, os dados apresentados com relação às facetas e aos domínios do WHOQOL-DIS-PD não deixam evidente uma vantagem significativa dos indivíduos praticantes de AF sobre os não praticantes. Porém, a média total mostra que as PCDv praticantes de AF são mais satisfeitas com a QV em comparação às não praticantes. 


\section{Considerações finais}

O levantamento empírico realizado com PCDv revela indícios de que a percepção da QV como um todo e algumas facetas e domínios que compõem os instrumentos de avaliação, podem ser influenciadas pela adesão à prática sistemática do goalball.

A compreensão mais abrangente sobre a influência da prática de AF na percepção da QV de PCDv dependerá de pesquisas que estendam a investigação para além das abordagens realizadas no presente estudo, o qual claramente apresenta limitações. Uma dessas limitações é sobre o tamanho da amostra pesquisada, a qual foi pequena e impediu que procedimentos estatísticos fossem realizados para confirmar a relação entre a prática de $\mathrm{AF}$ e a $\mathrm{QV}$, e a diferença entre os grupos. Ainda, aponta-se como limitação o fato de não terem sido levantadas informações específicas a respeito do estilo de vida dos participantes, o que poderia ajudar a entender melhor como essas PCDv se comportam em meio à sociedade, e, a partir disso, sustentar as explicações realizadas sobre as questões que contemplam a QV.

\section{Referências}

BOSCOLO, R. A.; SACCO, I. C.; ANTUNES, H. K.; MELLO, M. T.; TUFIK, S. Avaliação do padrão de sono, atividade física e funções cognitivas em adolescentes escolares. Revista Portuguesa de Ciências e Desporto, v. 7, n. 1, p. 18-25, 2007.

CANTORANI, J. R. H.; VARGAS, L. M.; REDKVA, P. E.; PILATTI, L. A.; GUTIERREZ, G. L. A dimensão da deficiência e o olhar a respeito das pessoas com deficiência a partir dos recenseamentos no Brasil. Revista Brasileira de Educação Especial, v. 21, n. 1, p. 159-176, 2015. crossef

CONFEDERAÇÃO BRASILEIRA DE DESPORTOS DE DEFICIENTES VISUAIS. Goalball. Disponível em:<http://cbdv.org.br/pagina/goalball>. Acesso em: 30 jan. 2015.

MASSOLA, R. M. Exercícios físicos na melhoria da qualidade de vida e capacidade de trabalho e na prevenção da dor e fadiga, entre trabalhadores de fábrica. 2007. $106 \mathrm{f}$. Dissertação (Mestrado em Educação Física) - Universidade Estadual de Campinas, Campinas, 2007.

METZNER, R. J.; FISCHER, F. M. Fadiga e capacidade para o trabalho em turnos fixos de doze horas. Revista Saúde Pública, v. 35, n. 6, p. 548-553, 2001. Crossef

NOCE, F.; SIMIM, M. A. M.; MELLO, M.T. A percepção de qualidade de vida de pessoas portadoras de deficiência física pode ser influenciada pela prática de atividade física? Revista Brasileira de Medicina do Esporte, v. 15, n. 3, p. 174-178, 2009. Crossef

OLIVEIRA, L. M. B. Cartilha do censo 2010: pessoas com deficiência. Brasília: SDH-PR/SNPD, 2012.

PEDROSO, B.; PILATTI, L. A.; GUTIERREZ, G. L.; PICININ, C. T. Cálculo dos escores e estatística descritiva do WHOQOL-bref através do Microsoft Excel. Revista Brasileira de Qualidade de Vida, v. 2. n. 1. p. 31-36, 2010. Crossef 
PEREIRA, E. F.; TEIXEIRA, C. S.; KOTHE, F.; MERINO, E. A. D.; DARONCO, L. S. Percepção de qualidade do sono e da qualidade de vida de músicos de orquestra. Revista Psiquiatria Clínica, v. 37 , n. 2, p. 48-51, 2010. Crossef

SCHERER, R. L.; LOPES, A. S. Atividade física habitual em adultos com deficiência visual: uma revisão sistemática. Pensar a Prática, v. 16, n. 1, p. 234-347, 2013. crossef

SILVA, M. L.; RUBIO, K. Superação no esporte: limites individuais ou sociais. Revista Portuguesa de Ciências do Desporto, v. 3, n. 3, p. 69-76, 2003.

STELLA, F.; GOBBI, S.; CORAZZA, D. I.; COSTA, J. L. R. Depressão no idoso: diagnóstico, tratamento e benefícios da atividade física. Motriz, v. 8, n. 3, p. 91-98, 2002.

TEIXEIRA, L. Exame de visão. Texto de apoio do curso de especialização, atividade física e saúde, 2010. Disponível em: <http://www.luzimarteixeira.com.br/wpcontent/uploads/2010/05/tabela-snellen-uso-e-interpretacao.pdf > . Acesso em: 10 set. 2014.

THE WHOQOL-DIS-GROUP. WHOQOL - Disabilities module manual. Genebra: World Health Organization, 2011.

THE WHOQOL GROUP. WHOQOL-bref: introduction, administration, scoring and generic version of assessment. Genebra: World Health Organization, 1996.

ZUCHETTO, A. T.; CASTRO, R. L. V. G. As contribuições das atividades físicas para a qualidade de vida dos deficientes físicos. Kinesis, v. 1, n. 26, p. 52-67, 2002. 